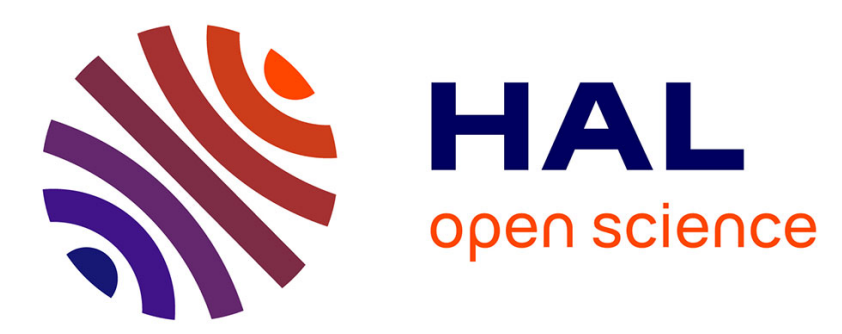

\title{
Sampling Semantic Data Stream: Resolving Overload and Limited Storage Issues
}

Naman Jain, Manuel Pozo, Raja Chiky, Zakia Kazi-Aoul

\section{To cite this version:}

Naman Jain, Manuel Pozo, Raja Chiky, Zakia Kazi-Aoul. Sampling Semantic Data Stream: Resolving Overload and Limited Storage Issues. First International Conference on Advanced Data and Information Engineering (DaEng 2015), Dec 2013, Kuala Lumpur, Malaysia. pp.41-48, 10.1007/978981-4585-18-7_5. hal-01314920

\author{
HAL Id: hal-01314920 \\ https://hal.science/hal-01314920
}

Submitted on 12 May 2016

HAL is a multi-disciplinary open access archive for the deposit and dissemination of scientific research documents, whether they are published or not. The documents may come from teaching and research institutions in France or abroad, or from public or private research centers.
L'archive ouverte pluridisciplinaire HAL, est destinée au dépôt et à la diffusion de documents scientifiques de niveau recherche, publiés ou non, émanant des établissements d'enseignement et de recherche français ou étrangers, des laboratoires publics ou privés.

$$
\text { Copyright }
$$




\title{
Sampling Semantic Data Stream: Resolving Overload and Limited Storage Issues
}

\author{
Naman Jain ${ }^{1}$, Manuel $\mathrm{Pozo}^{2}$, Raja $\mathrm{Chiky}^{2}$, and Zakia Kazi-Aoul ${ }^{2}$ \\ 1 VIT University, Vellore, TN 632014, India \\ namanjain2009@vit.ac.in \\ 2 ISEP - LISITE, Paris 75006, France \\ \{manuel-jesus.pozo-ocana, raja.chiky, zakia.kazi\}@isep.fr
}

\begin{abstract}
The Semantic Web technologies are being increasingly used for exploiting relations between data. In addition, new tendencies of realtime systems, such as social networks, sensors, cameras or weather information, are continuously generating data. This implies that the data and the links between them are becoming extremely vast.

Such huge quantity of data needs to be analyzed, processed, as well as stored, if necessary. In this paper, we propose sampling operators that allow us to drop RDF Triples from the incoming data. Thereby, helping us to reduce the load on existing engines like CQELS, C-SPARQL, which are able to deal with big and linked data. Hence, the processing efforts, time as well as required storage space will reduce remarkably.

We have proposed Uniform Random Sampling, Reservoir Sampling and Chain Sampling operators which may be implemented depending on the application.
\end{abstract}

Keywords: Big Data, Linked data-stream, Processing time, Sampling

\section{Introduction}

The semantic web handles many systems, such us Twitter, Facebook or Google, which generate increasing volumes of semantic data everyday. The problem of "too much (streaming) data but not enough (tools to gain and derive) knowledge" was tackled by [7]. They envisioned a Semantic Sensor Web (SSW), in which sensor data are annotated with semantic metadata to increase interoperability and provide contextual information essential for situational knowledge. CQELS[6], SPARKWAVE[5], C-SPARQL[3] etc. are existing technologies to exploit these semantic and streaming (continuous and infinite) data, and are based on recommended standard RDF, as the format of representation.

CQELS[6] is a native approach in an RDF environment based on 'whiteboxes'. It provides its own processing model and its own operators to deal with streams, for example, window operators or query semantic operators. CSPARQL[3] on the other hand, uses a 'black-box' approach which delegates the processing to other engines such as stream/event processing engines and SPARQL query processors by translating to their provided languages. 
Although almost all the engines are based on the SPARQL Language, there are only a few systems which are able to process big quantity of data on the fly. Moreover, these engines do not feature any tool that would allow them to reduce the processing efforts and improve the processing time. For many applications, we must obtain compact summaries of the stream. These summaries could allow accurate answering of queries with estimates, which approximate the true answers over the original stream [4].

Thus, we propose the implementation of such sampling operators that could be used in conjunction with other existing real-time engines. These sampling operators will allow us to deal with the requested population by applying heuristic methods. Uniform Random Sampling, Reservoir Sampling and Chain Sampling were implemented on the data streams and were then compared with the error percentage, storage requirements and the load suffered by the engine. Thus, these sampling methods will help reduce processing time and the required memory space.

\section{Extension to Existing Systems}

We propose to extend existing semantic data stream querying engines by creating an external abstraction of the sampling operator. The extension acts as follows:

First, we recognize the different operators of the language and split the initial query according to them. This will allow us to use the same operator at different levels of the query: in the input, for sampling static data or streaming data and in the output to sample the result of the query, or both simultaneously.

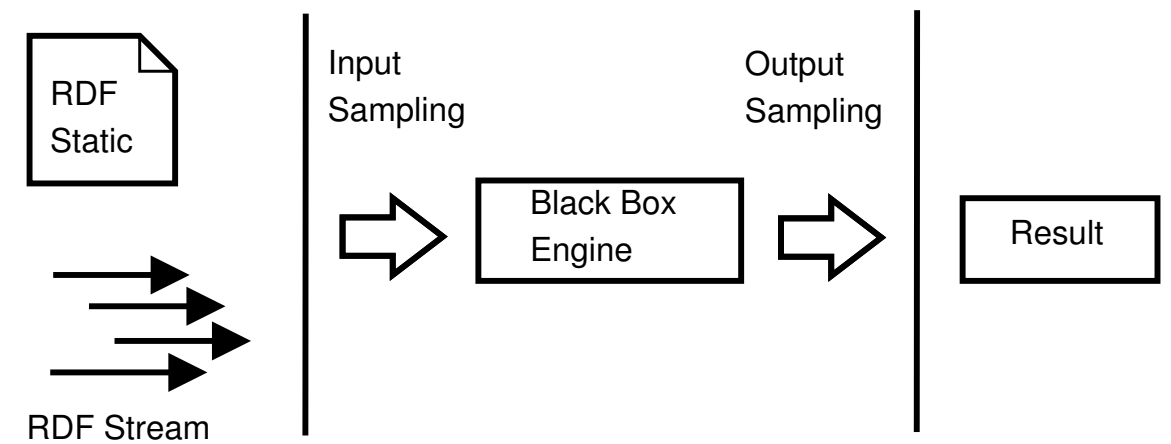

Fig. 1. Block Diagram

After splitting the query, we make syntax correction. If we identify any errors in our operator, we stop the execution of the query. Hence, if everything is correct, we create a key-value map where we store all the resources and all the requested sampling actions. Thus, we can apply the sampling operator independently by creating a thread for each of them. 
We start the abstraction of the operator by taking out all the sampling instances in the query. This will allow us to avoid the correction of the rest of operators and leave the task to the specific engine itself.

When we send this query to the engine, as shown in Figure 1, it will run all the threads in charge of each source. By adapting those threads, we apply the sampling method just before the data comes to the engine, thus discharging the processing tasks. In a similar way, we adapt the output of the engines, so as to apply sampling methods at the output and lighten storage space.

\section{Sampling Methods}

The query would contain the information of sampling type and the sampling percentage for each unique stream. We apply the appropriate sampling method for each stream in different threads. We used Uniform Random Sampling, Reservoir Sampling and Chain Sampling to compare their advantages and disadvantages. We may choose an appropriate sampling method depending on the application used for.

We have implemented our sampling operators with CQELS[6] and C-SPARQL [3] engine. Example below shows simple type of sampling at $50 \%$ i.e. taking one triple and dropping the next one, using CQELS as:

PREFIX lv: <http://deri.org/floorplan/>

SELECT ?person ?locName

FROM NAMED <C:/floorplan.rdf>

WHERE \{

STREAM <C:/rfid.stream> [NOW] [SAMPLING \%50]

\{?person lv:detectedAt ?loc \}

GRAPH <C:/floorplan.rdf> \{?loc lv:name ?locName $\}$ \}

[OUTSAMPLING \%80]

We implemented Output Sampling using

Operator: [OUTSAMPLING \% \{Sampling Percentage $\}$ ]

As shown in Figure 1, the output of the engine goes through this operator and is given as final result only if the operator permits. Normal sampling algorithm has been used for this i.e. if $\% 80$ is specified, then only 4 out of 5 results are termed as final results. If Output Sampling operator is not specified, then the percentage is assumed to be 100 and no result is left out after the processing is done by the engine.

The sampling operators can also be implemented on similar systems like Sparkwave[5], ETALIS[1] after changing few configurations. In the examples to follow CQELS is used for the experimentation.

For analysing the sampling methods, we computed average of 8-hour daily maximum ozone concentrations as measured by Clean Air Status and Trends 
Network (CASTNET) in United States of America. The RDF data contains $2,159,133$ triples consisting of 308,380 number of entries. ${ }^{3}$

\subsection{Uniform Random Sampling}

In this sampling method, incoming triples are sent to the engine, only if uniform random generation of true/false, with a probability equal to sampling percentage divided by 100 , returns true.

Operator: [UNISAMPLING \%\{Sampling Percentage $\}$ ]

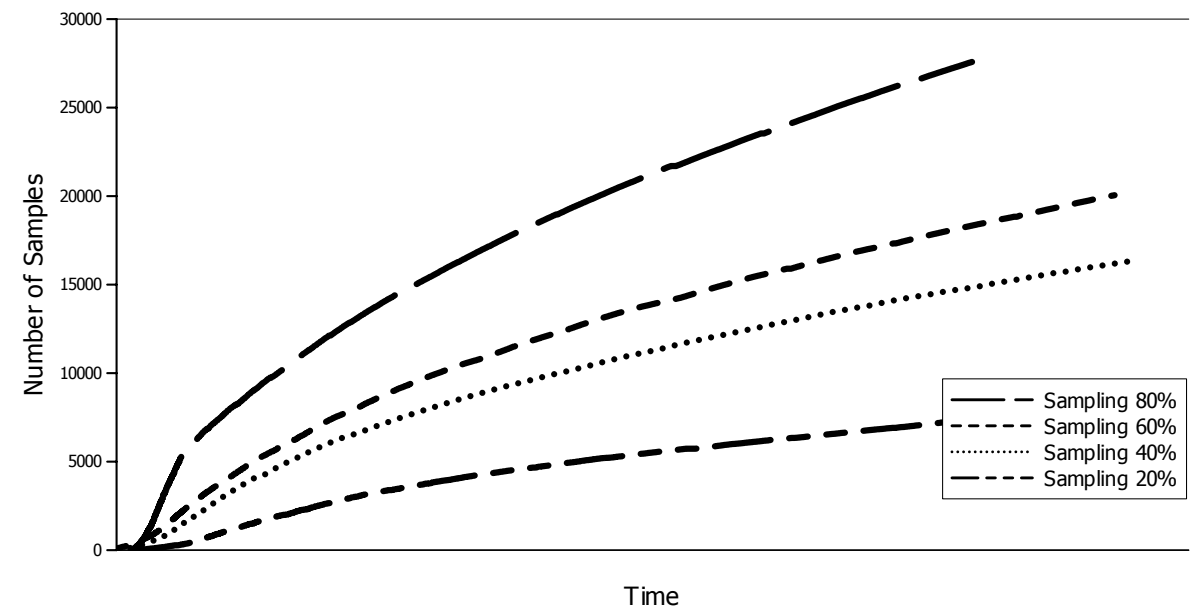

Fig. 2. Sample size variation/ Number of triples processed by engine in Uniform Random Sampling (During time span of 1 minute)

For instance, if the input sampling percentage is set to 60 , then we find the probability by dividing 60 with 100 which is 0.6 . Then, we randomly generate boolean values with this probability and send the triples to the engine if the boolean value returned is true. Thereby reducing the processing load and time of the engine. This helps to keep number of samples in proportion to total incoming data. The query ${ }^{4}$ is of the form

WHERE \{

STREAM <C:/data-8.stream> [NOW] [UNISAMPLING \%60]

\{?location vocab:ozone_8hr_daily_max ?value\} \}

Figure 2 shows sample size variation and number of triples processed by the engine. They both are same in this type of sampling as there is no removal of

\footnotetext{
${ }^{3}$ http://data-gov.tw.rpi.edu/wiki/Dataset_8/

${ }^{4}$ PREFIX \& SELECT operators are omitted here to avoid repetition
} 
elements from the sample. This sampling method is less preferred as the sample size keeps on growing, which may lead to shortage of storage space, and even the outdated data element will be existing.

\section{$3.2 \quad$ Reservoir Sampling}

The problem of maintaining a sample of specified size ' $\mathrm{k}$ ' is overcome by Reservoir Sampling [8]. In this, we add the triple 'i' to the sample with probability k/i and discarding a randomly chosen element from the reservoir or sample to make room for the new element.

Operator: [RESSAMPLING \{Reservoir Size\}]

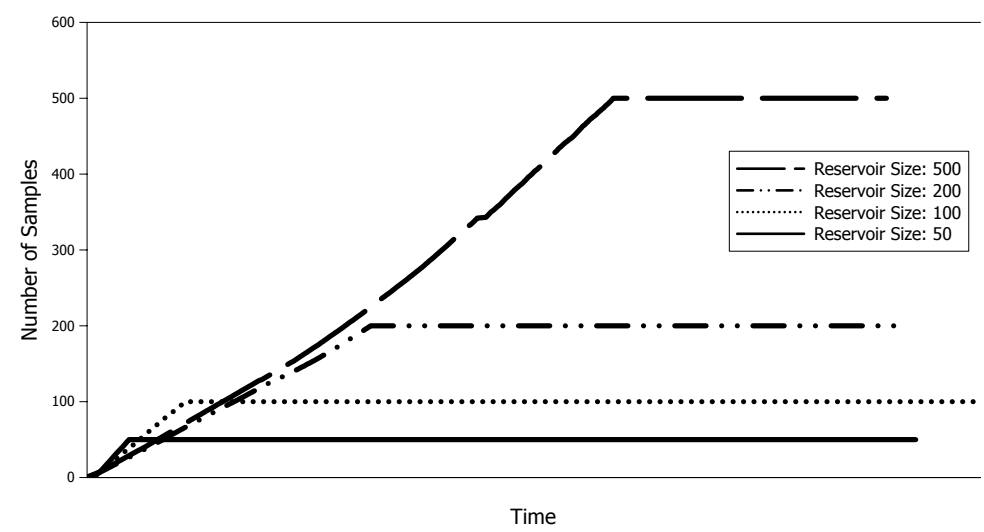

(a) Sample Size variation after using Reservoir Sampling

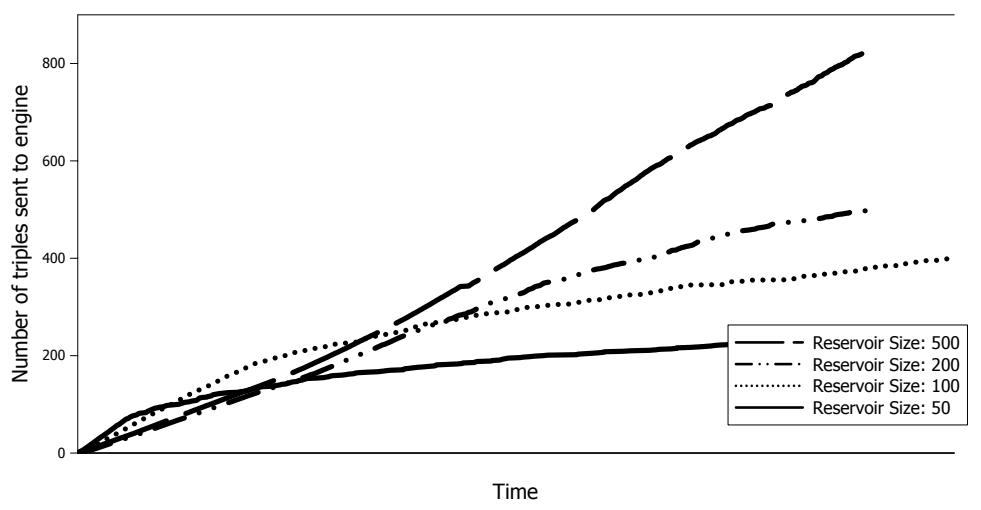

(b) Load on engine after using Reservoir Sampling

Fig. 3. Plot of sample size variation and load on engine after using Reservoir Sampling (All plots are for the same time duration of 1 minute) 
For instance, here reservoir size of 500 ensures that the number of samples at any point may not exceed 500. Initially, triples are added to the reservoir until it is full. Then incoming triples, with stream number ' $i$ ' are added with probability 500/i, after replacing a randomly chosen element from the reservoir. We can also say that chances of adding a triple reduces as the stream number increases. Query ${ }^{4}$ is

\section{WHERE \{}

STREAM <C:/data-8.stream> [NOW] [RESSAMPLING 500]

\{?location vocab:ozone_8hr_daily_max ?value\}

Figure 3a shows that the sample size becomes constant after the reservoir is full and Figure 3b shows number of triples processed by the engine over the time. This sampling method helps us to limit the maximum storage space of the samples, but is not favourable when recent data is more important to us, as the sample data may have 'expired'. A significant reduction in load on the engine can also be noted in Figure 3b when compared to Figure 2.

\subsection{Chain Sampling}

In [2] authors state the Chain Sampling method for sequence based windows. In this method, if the window size is ' $n$ ' we add each new element ' $i$ ' in the sample with probability $\min (\mathrm{i}, \mathrm{n}) / \mathrm{n}$. As each element is added to the sample we choose an index, in the range $(i+1, i+n)$, of the element that replaces it when it expires. We choose this range as when element ' $i$ ' expires the window would be in the range $(i+1, i+n)$. Once the element with that index arrives, we store it and choose the index which replaces it when it expires, thus forming a chain of replacements. STEP operator is used to specify the size of steps that the sequence based window takes. Operator: [CHNSAMPLING\{WindowSize\} STEP $\{$ StepSize $\}]$

and the query ${ }^{4}$ is of the form

WHERE \{

STREAM <C:/data-8.stream> [CHNSAMPLING 500 STEP 2]

\{?location vocab:ozone_8hr_daily_max ?value\} \}

The above query shows that window size ' $\mathrm{n}$ ' is 500 and window moves in steps of 2. Figure 4a shows that the sample size increases as the window size is increased but Figure 4b shows that number of triples being processed by the engine is lower for higher window sizes. We also note from Figure $3 \mathrm{~b}$ and Figure $4 \mathrm{~b}$ that load on the engine is much higher for Chain Sampling.

Chain Sampling ensures to keep sample elements which are present only in the window and eliminates the problem of 'expired element' in the sample. But, the act of storing replacements does not reduce the memory space requirements and load on the engine as effectively as Uniform Random Sampling and Reservoir Sampling. 


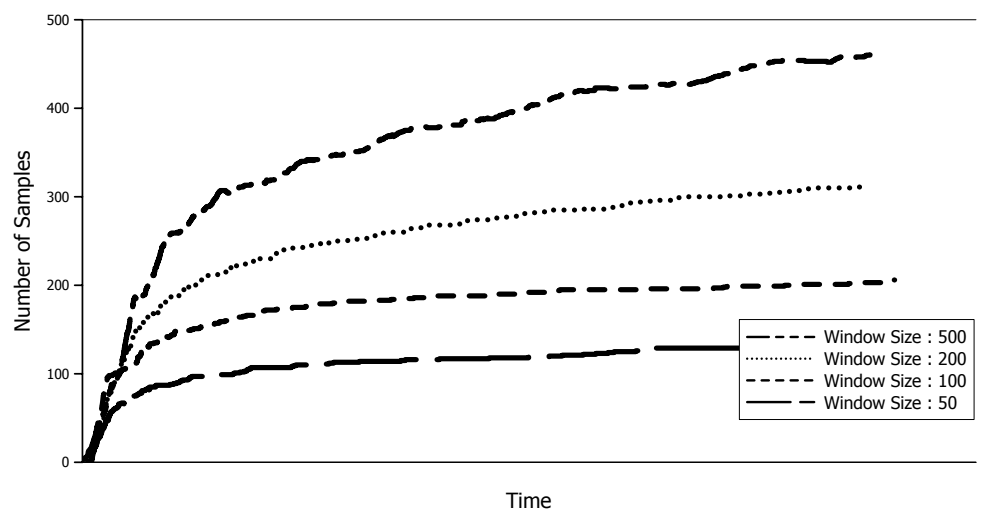

(a) Sample Size variation after using Chain Sampling

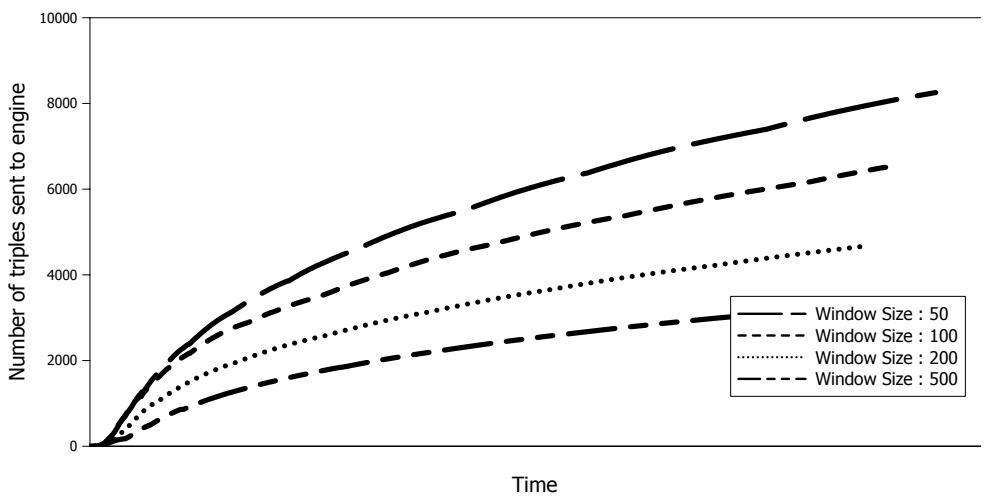

(b) Load on engine after using Chain Sampling

Fig. 4. Plot of sample size variation and load on engine after using Chain Sampling (All plots are for the same time duration of 1 minute)

\section{Experimentation}

The queries in Section 3 were first executed without any sampling, and then after incorporating different sampling operators. After computing 50,000 entries of 8-hour daily maximum ozone concentrations, without any sampling operator, their average was found to be $\mathbf{4 9 . 9 1 6 1}$. Also, the average of results obtained by 20 iterations (after applying each sampling operator) is demonstrated in Table 1. All results are for 50,000 entries.

We may note that in Uniform Random Sampling probability of error reduces as the sampling percentage is increased while load on the engine is in coalition with the sampling percentage specified. In Reservoir Sampling, we see that the probability of error reduces with increase in reservoir size and a significant reduction in sample size as well as load on the engine is observed. 
Table 1. Variation of Sample size, Load on engine and Error due to different sampling methods

\begin{tabular}{|c|c|c|c||c|c|c|}
\hline Method & $\begin{array}{c}\text { Para- } \\
\text { meter }\end{array}$ & $\begin{array}{c}\text { Sample } \\
\text { Size }\end{array}$ & $\begin{array}{c}\text { Triples } \\
\text { Processed }\end{array}$ & $\begin{array}{c}\text { Effective } \\
\text { Sampling \% }\end{array}$ & $\begin{array}{c}\text { \% Triples } \\
\text { Processed }\end{array}$ & $\begin{array}{c}\text { \% of } \\
\text { Error }\end{array}$ \\
\hline Uniform & 20 & 10034 & 10034 & 20.06 & 20.06 & 0.1819 \\
Random & 40 & 19993 & 19993 & 39.98 & 39.98 & 0.1540 \\
Sampling & 60 & 29998 & 29998 & 59.99 & 59.99 & 0.0793 \\
[Sampling \%] & 80 & 40002 & 40002 & 80.00 & 80.00 & 0.0494 \\
\hline & 500 & 500 & 2807 & 1.00 & 5.61 & 1.3532 \\
Reservoir & 1000 & 1000 & 4925 & 2.00 & 9.85 & 0.8041 \\
Sampling & 2000 & 2000 & 8445 & 4.00 & 16.89 & 0.6047 \\
[Reservoir Size] & 5000 & 5000 & 16490 & 10.00 & 32.98 & 0.3123 \\
\hline & 10000 & 10229 & 42589 & 20.45 & 85.17 & 0.4410 \\
Chain & 20000 & 13183 & 36332 & 26.36 & 72.66 & 0.5774 \\
Sampling & 30000 & 14857 & 31260 & 29.71 & 62.52 & 2.5181 \\
[Window Size] & 40000 & 16116 & 27298 & 32.23 & 54.59 & 0.6559 \\
\hline
\end{tabular}

In Chain Sampling percentage of error depends on the elements in the current window and an improvement in sample size is observed in comparison to Uniform Random Sampling but load on the engine is comparatively high as sample replacements are also required to be processed by the engine.

\section{Conclusion}

The growing generated data from web applications is becoming a problem for the processing systems, and the relation between data is causing troubles when attempting to exploit data repositories. Therefore, In this paper we have proposed an extension of a real-time request system that allow us to reduce processing tasks and memory space requirements.

Different sampling methods suggested are useful for different applications. For example, if accuracy is required then we may go for Uniform Random Sampling with appropriate sampling percentage, and if memory size is fixed, we should go for Reservoir Sampling. Chain Sampling with suitable window size is favourable only when the samples need to be 'new'. In near future, we will be building the sample according to the importance of incoming data elements, rather than randomly choosing them.

\section{References}

1. D. Anicic, S. Rudolph, P. Fodor, and N. Stojanovic. Stream reasoning and complex event processing in etalis. Semantic Web, 3(4):397-407, 2012.

2. B. Babcock, M. Datar, and R. Motwani. Sampling from a moving window over streaming data. In Proceedings of the thirteenth annual ACM-SIAM symposium on 
Discrete algorithms, SODA '02, pages 633-634. Society for Industrial and Applied Mathematics, 2002.

3. D. F. Barbieri, D. Braga, S. Ceri, E. Della Valle, and M. Grossniklaus. C-sparql: Sparql for continuous querying. In Proceedings of the 18th international conference on World wide web, pages 1061-1062. ACM, 2009.

4. E. Cohen, G. Cormode, and N. Duffield. Structure-aware sampling on data streams. In Proceedings of the ACM SIGMETRICS joint international conference on Measurement and modeling of computer systems, pages 197-208. ACM, 2011.

5. S. Komazec, D. Cerri, and D. Fensel. Sparkwave: continuous schema-enhanced pattern matching over rdf data streams. In Proceedings of the 6th ACM International Conference on Distributed Event-Based Systems, pages 58-68. ACM, 2012.

6. D. Le-Phuoc, M. Dao-Tran, J. X. Parreira, and M. Hauswirth. A native and adaptive approach for unified processing of linked streams and linked data. In The Semantic Web-ISWC 2011, pages 370-388. Springer, 2011.

7. A. Sheth, C. Henson, and S. S. Sahoo. Semantic sensor web. Internet Computing, IEEE, 12(4):78-83, 2008.

8. J. S. Vitter. Random sampling with a reservoir. ACM Transactions on Mathematical Software (TOMS), 11(1):37-57, 1985. 